\title{
CORRECTION
}

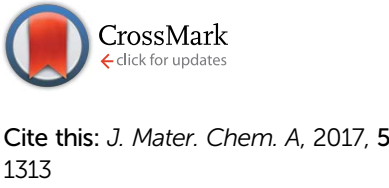

DOI: 10.1039/c6ta90259f

www.rsc.org/MaterialsA

\section{Correction: Robust liquid-repellent coatings based on polymer nanoparticles with excellent self- cleaning and antibacterial performances}

Yuchen Fu, Jingxian Jiang, Qinghua Zhang, ${ }^{*}$ Xiaoli Zhan and Fengqiu Chen

Correction for 'Robust liquid-repellent coatings based on polymer nanoparticles with excellent selfcleaning and antibacterial performances' by Yuchen Fu et al., J. Mater. Chem. A, 2017, 5, 275-284.

The weight ratio BMA/HEMA/TFOA/QDEMA of FC3 in Table 1 should be altered from "7/1/8/6" to "5/1/8/6".

The Royal Society of Chemistry apologises for these errors and any consequent inconvenience to authors and readers. 